\title{
Berberine Improves the Symptoms of DHEA-Induced PCOS Rats by Regulating Gut Microbiotas and Metabolites
}

\author{
Hao-Ran Shen ${ }^{\mathrm{a}}$ Xiao Xu $\mathrm{Xu}^{\mathrm{a}}$ Dan Ye ${ }^{\mathrm{a}}$ Xue-Lian Lia,b \\ aDepartment of Gynecology, Obstetrics \& Gynecology Hospital of Fudan University, Shanghai, China; \\ bShanghai Key Laboratory of Female Reproductive Endocrine-Related Diseases, Shanghai, China
}

\section{Keywords}

Polycystic ovary syndrome $\cdot$ Insulin resistance $\cdot$ Gut microbiota Berberine

\begin{abstract}
Objectives: The aim of this study was to investigate the effects of berberine on polycystic ovary syndrome (PCOS) with insulin resistance (IR). Design: This study performed 16S rRNA sequencing and metabolomic analysis on dehydroepiandrosterone (DHEA)-induced PCOS rats treated with berberine, focusing on the improvement of PCOS-IR by modifying gut microbiota and metabolism. Methods: Forty-two female Sprague Dawley rats were randomly divided into 4 experimental groups of 8 rats each (PCOS + HFD, PCOS + HFD + BBR, $\mathrm{NCD}+\mathrm{PCOS}$, and NCD + PCOS + BBR groups). Homeostasis model assessment of insulin resistance (HOMA-IR) index-related indicators and hormone level in serum were analyzed. $16 \mathrm{~S}$ rRNA sequencing and metabolomic analysis were performed on DHEA-induced PCOS rats treated with berberine. In addition, the differential microbiotas and metabolites were screened. Also, enrichment analysis was carried out on the differential metabolites. Finally, we constructed a correla-
\end{abstract}

tion network to analyze the correlation between differential microbiotas and metabolites. Results: Firmicutes and Bacteroidetes were changed at the phylum level, and Romboutsia, Bacteroides, and Clostridium_sensu_stricto_ 1 were changed at the genus level after berberine treatment. In addition, a total of 26 differential operational taxonomic units and 3 metabolites (glutamine, unsaturated acids $[\mathrm{CH}=\mathrm{CH}]$, and glucose) between 2 groups were obtained. Moreover, these metabolites were mainly involved in type 2 diabetes mellitus, 2-component system, and ABC transporter Kyoto Encyclopedia of Genes and Genomes pathways. And, 3 microbiotas (Lachnospiraceae_NC2004_group, Flavonifractor, and Parasutterella) were regulated by glucose and glutamine. Limitations: The sample size involved in this study is relatively small. In addition, relevant experiments need to be performed to verify the obtained results from this study, and indepth functional studies are needed. Conclusions: Berberine is effective in improving the pathological condition in PCOS by regulating the gut microbiotas and metabolites. This study will provide evidence for therapeutic efforts to treat PCOS-IR using berberine.

(C) 2021 The Author(s) Published by S. Karger AG, Basel
(C) 2021 The Author(s)

Published by S. Karger AG, Basel

This is an Open Access article licensed under the Creative Commons Attribution-NonCommercial-4.0 International License (CC BY-NC) (http://www.karger.com/Services/OpenAccessLicense), applicable to the online version of the article only. Usage and distribution for commercial purposes requires written permission.
Correspondence to:

Hao-Ran Shen, alainshen@aliyun.com

Xue-Lian Li, xllifc@fudan.edu.cn 


\section{Introduction}

Polycystic ovary syndrome (PCOS), also known as Stein-Leventhal syndrome, was first reported in 1935 [1]. PCOS is the most common endocrine and metabolic disorder in adolescent women of reproductive age, with an incidence of $5-10 \%$ [2-4]. Moreover, PCOS is a significant cause of infertility [5]. It is generally believed that insulin resistance (IR), which is an increased risk of glucose intolerance and type 2 diabetes, is the core of PCOS, and there are about $50-75 \%$ of PCOS patients with different degrees of IR [6, 7].

Insulin-sensitizing agents can improve the endocrine and clinical symptoms of PCOS patients in some way. Metformin is a widely used insulin-sensitizing agent, which was firstly administered in obese PCOS women in 1994 to reduce serum levels of insulin and androgen and regularize the cycle of menses [8], but some patients worry about the potential adverse effects [9]. Berberine is an isoquinoline alkaloid isolated from the traditional Chinese medicines coptis and phellodendron $[10,11]$, and it exerts beneficial effects that are hypoglycemic and lipid lowering, ultimately improving IR [12, 13]. Lublinski et al. [14] suggested that berberine can treat metabolic disorders such as IR by improving gut microbiota, and $\mathrm{Wu}$ et al. [15] found that berberine can be used to treat PCOS.

The gut microbiota entails microorganisms that exist in the human gastrointestinal tract, reaching approximately $10^{13}-10^{14}$, with about 5001,000 species [16]. It is interesting to explore the role of gut microbiota in PCOS, as the androgen level in PCOS women is always elevated [17]. Thus, we hypothesize that excess androgen biosynthesis in PCOS may result in the dysbiosis of host gut microbiota, and modulating of gut microbiota may be beneficial for PCOS treatment. In the present study, we performed 16S rRNA sequencing and metabolomic analysis on dehydroepiandrosterone (DHEA)-induced PCOS rats treated with berberine, focusing on the improvement of PCOS-IR by modifying gut microbiota and metabolism. Through this work, we hope to provide evidence that berberine has the therapeutic effect to treat PCOS-IR.

\section{Materials and Methods}

\section{Animals and Model}

Forty-two female Sprague Dawley rats (21 days of age) were randomly divided into 4 experimental groups of 8 rats each (PCOS $+\mathrm{HFD}, \mathrm{PCOS}+\mathrm{HFD}+\mathrm{BBR}, \mathrm{NCD}+\mathrm{PCOS}$, and NCD + PCOS $+\mathrm{BBR}$ groups). Two control groups were composed of 5 rats each
( $\mathrm{NCD}$ and $\mathrm{NCD}+\mathrm{BBR}$ groups). The experimental groups were continuously injected with $6 \mathrm{mg} / 100 \mathrm{~g} /$ day of body weight of DHEA (source leaf organisms, S24516) in $0.2 \mathrm{~mL}$ of soybean oil for 21 days, while the control groups were only injected with 0.2 $\mathrm{mL}$ of soybean oil. Partial PCOS model rats were fed with a highfat diet (HFD) to induce IR, and the control groups were fed with a normal chow diet (NCD) (the nutrient ratios in the HFD included $10 \%$ lard, $20 \%$ sucrose, $2.5 \%$ cholesterol, $1 \%$ porcine bile salt, $1 \%$ egg, $30 \%$ sprouts, and $35.5 \%$ basic feed, obtained from the Shanghai Laboratory Animal Center [SLAC], Shanghai, China). Then, the rats in NCD + BBR, PCOS + HFD + BBR, and NCD + PCOS + BBR groups were treated with berberine of $99 \%$ purity $(150 \mathrm{mg} / \mathrm{kg})$ (Sigma-Aldrich, Louis, MO, USA) once daily for 6 weeks.

\section{Homeostasis Model Assessment of Insulin Resistance Index-}

Related Indicators

Blood samples from 3 rats in every group were taken from the tail after an overnight fasting $(8 \mathrm{~h})$, to measure fasting plasma glucose by using an ACCU-CHEK Performa glucose [18] meter (Roche Diabetes Care, Indianapolis, IN, USA) and fasting insulin (FINS) by using a Rat Insulin ELISA Kit (Thermo Scientific, Rockford, IL, USA) according to the manufacturer's instructions. The HOMA-IR was calculated as (fasting plasma glucose $[\mathrm{mmol} / \mathrm{L}] \times$ FINS $[\mu \mathrm{IU} / \mathrm{mL}]) / 22.5$, and higher HOMA-IR values indicated lower insulin sensitivity [18].

\section{Detection of the Hormone Level in Serum}

A total of 3 rats in every group were used to detect the hormone level in serum. The level of testosterone in rats was determined by ELISA, and the Testosterone Parameter Assay Kit (KGE010; R\&D systems) was used to measure the testosterone level according to the manufacturer's instruction.

\section{Bacterial Sequencing and Analysis}

This study aimed to evaluate the effects of berberine on PCOSIR, since HFD induces significant metabolic disorders and gut microbiome dysbiosis in PCOS $[19,20]$. Thus, 16 S rRNA sequencing and metabolomic analysis were performed on the PCOS + HFD + BBR and PCOS + HFD groups to exclude the influence factor of HFD. Fecal samples were collected from the rats in the 2 groups, and the DNeasy PowerSoil Kit (Qiagen, Germantown, MD, USA) was used to extract the bacterial genomic DNA. The V3-V4 region of the $16 \mathrm{~S}$ rRNA gene was selected for subsequent pyrosequencing, and the acquired gene sequences were PCR amplified with barcoded universal primers. Then, the PCR products were visualized on a 2\% agarose gel and quantified with a Qubit fluorometer (Promega, Madison, WI, USA).

Data of $16 \mathrm{~S}$ sequencing have been uploaded to the NCBI database (SRA accession: PRJNA594263, https://www.ncbi.nlm.nih. gov/sra/PRJNA594263). The sequencing data were preprocessed to generate high-quality sequences, and the Vsearch software was used to combine the sequences according to their similarity. The remaining sequences with a similarity $\geq 97 \%$ were grouped into the same operational taxonomic unit (OTU). Then, differential expression analysis was performed using the quasi-likelihood $F$ test of the edgeR package with the cutoff value of $p$ value $<0.05$. And, the Pearson correlation coefficient of the differential gut bacterial microbiome was calculated with a threshold of $p$ value $<0.05$ and $|r|>0.8$. 
a

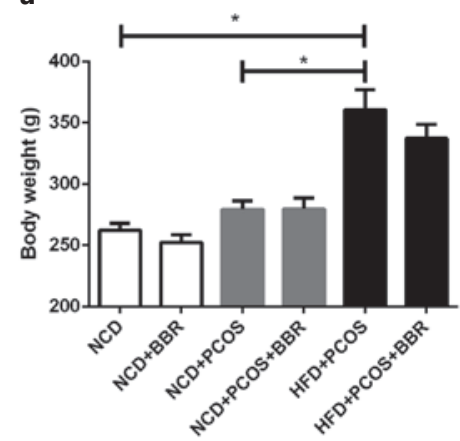

d

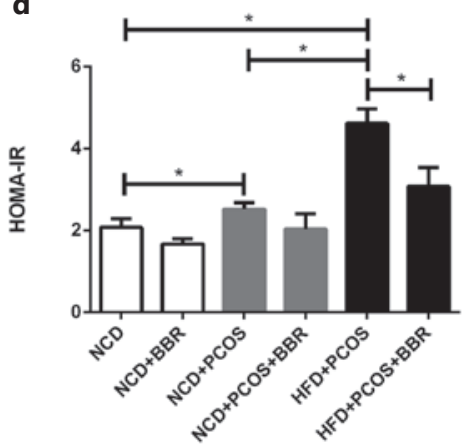

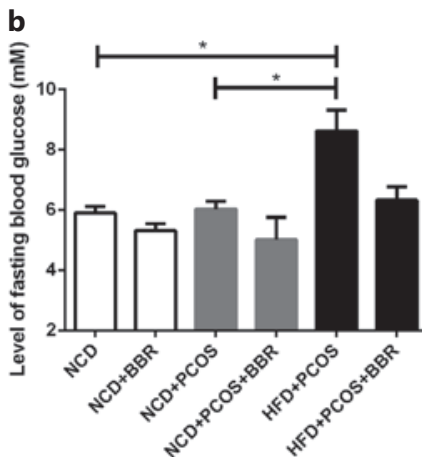

e

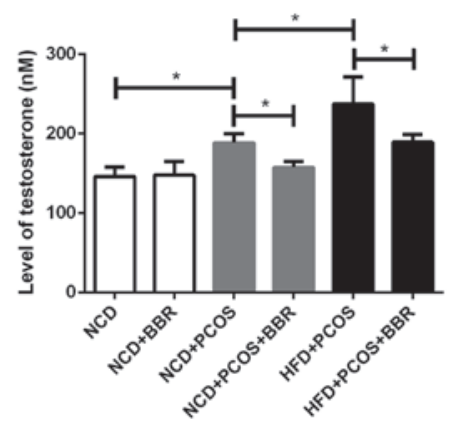

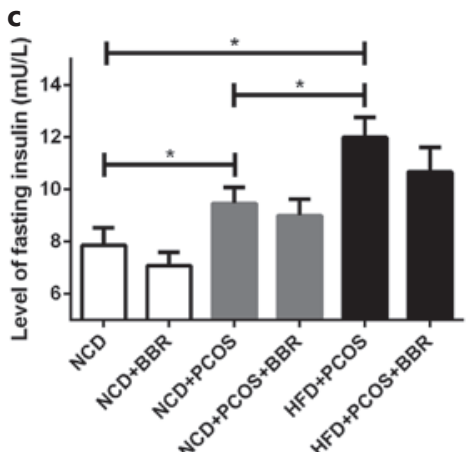

Fig. 1. Berberine treatment altered IR-related indices and testosterone level in the PCOS rats according to our previous study [21]. Body weight (a); level of fasting blood glucose (b); level of fasting insulin (c); HOMA-IR (d); level of testosterone $(\mathbf{e}) .{ }^{*} p$ value $<0.05$. IR, insulin resistance; PCOS, polycystic ovary syndrome; HOMA-IR, homeostasis model assessment of insulin resistance.

\section{Metabolomic Analysis}

Metabolomic analysis was performed on the PCOS + HFD + $\mathrm{BBR}$ and PCOS + HFD groups. Blood samples were collected from rats in the 2 groups, and the serum was separated by centrifugation $\left(13,000 \mathrm{rpm}, 10 \mathrm{~min}\right.$, and $\left.4^{\circ} \mathrm{C}\right)$ and stored at $-80^{\circ} \mathrm{C}$. Then, the LCMS analysis was conducted by ProfLeader Biotech Co., Ltd. (Shanghai, China).

Data of metabolomic analysis have been uploaded to a public online database (https://figshare.com/s/1463481d11efafc88b97, DOI: $10.6084 / \mathrm{m} 9$.figshare.11662473). LC-MS raw data were collected using the UNIFI software and processed using Proqenesis QI software. The Proqenesis QI analysis provided a matrix including peak picking, peak grouping, retention time (RT) correction, second peak grouping, and annotation of isotopes for every sample. Any peak detected in $<50 \%$ of quality control samples or $80 \%$ of biological samples was removed; the combination of the RT and $\mathrm{m} / \mathrm{z}$ data was used to identify each ion. Then, the data were preprocessed by normalization, and the principal component analysis was performed by prcomp function to assess the outlier detection and batch effect. The ropls in R package (http://bioconductor.org/ packages/release/bioc/html/ropls.html, Version 1.6.2) was used to carry out orthogonal partial least-squares discriminant analysis (OPLS-DA) and PLS-DA to visualize the metabolic alterations among the 2 groups. And, the variable importance in the projection (VIP) value was calculated. Moreover, the differential metabolites were screened with the cutoff value of $p$ value $<0.05$ and VIP $>1$. Besides, enrichment analysis was conducted using MBROLE (http://csbg.cnb.csic.es/mbrole2/analysis.php) with the threshold of $p$ value $<0.05$.

\section{Correlation Analysis between Differential Microbiotas and \\ Metabolites}

The Pearson correlation coefficients of differential microbiotas and differential metabolites were calculated, and the correlation network was constructed through Cytoscape software (https://cytoscape.org/).

\section{Statistical Analysis}

All statistical analyses were performed using SPSS statistical software package standard version 16.0 (SPSS, Inc., Chicago, IL, USA) and GraphPad prism 5 (GraphPad Software, San Diego, CA, USA). The data are presented as mean \pm standard deviation. Student's $t$ test was used to analyze the differences between 2 groups, and the differences between individual OTUs were analyzed by the nonparametric Kruskal-Wallis test. $p$ value $<0.05$ was considered statistically significant. 


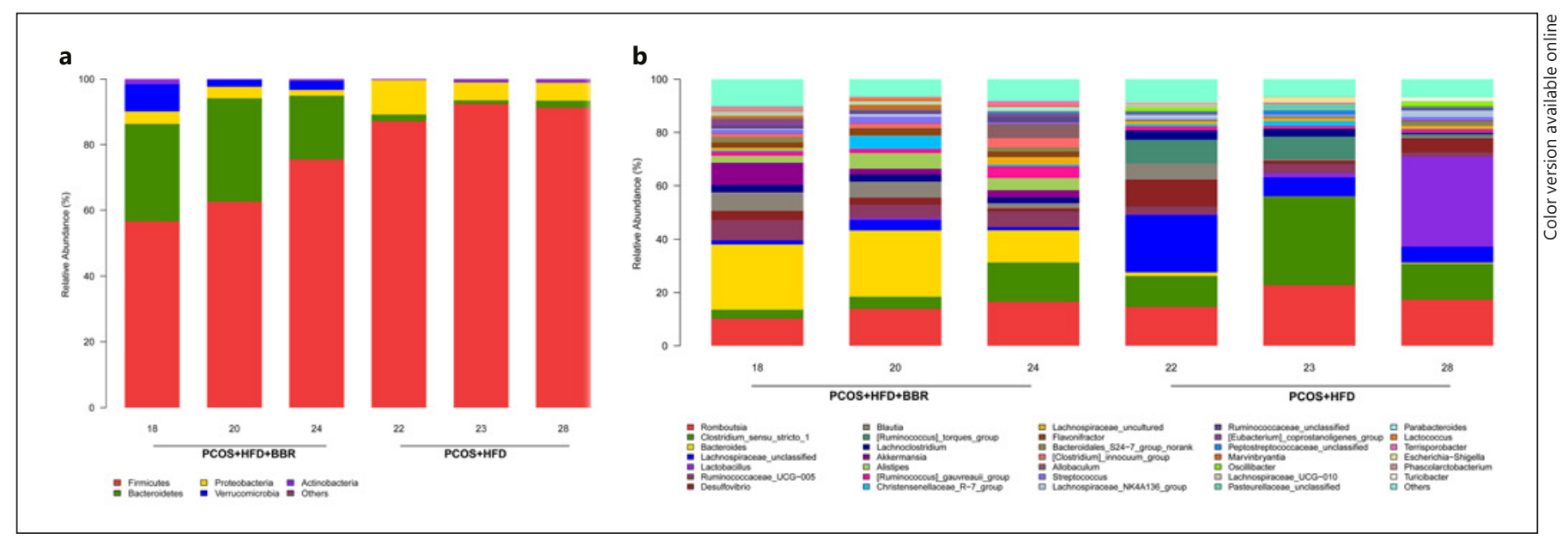

Fig. 2. Berberine alters microbiota composition in PCOS rats. At the phylum level (a); at the genus level (b). PCOS, polycystic ovary syndrome.

\section{Results}

\section{Berberine Treatment Altered IR-Related Indices and Testosterone Level in the PCOS Rats}

Herein, the IR-related indices, testosterone level, and ovarian morphology changes were evaluated according to our previous study [21]. As shown in Figure 1a-d, the body weight, fasting blood glucose, fasting insulin, and HOMA-IR index in the HFD group were higher than those in NCD groups, indicating that the rats in the HFD group had IR. However, the HOMA-IR index of the PCOS + HFD + BBR group was significantly lower than that of the PCOS + HFD group ( $p$ value $<0.05$ ), and the other 3 indicators also showed a downward trend, although there was no significant difference. In addition, the level of testosterone in NCD + PCOS + BBR and HFD + PCOS + $\mathrm{BBR}$ groups was significantly decreased than that of HFD + PCOS and NCD + PCOS groups, respectively ( $p$ value $<0.05)$ (Fig. 1e). Taken together, these data demonstrate that berberine has the potential to alter PCOS testosterone level and IR values in PCOS model rats.

\section{Berberine-Induced Changes in the Gut Bacterial Microbiome}

Furthermore, the taxon summary indicated distinct changes in the gut microbial composition in response to berberine administration. In terms of the assignment at the phylum level, the dominant phyla were Firmicutes and Bacteroidetes (Fig. 2a). At the genus level, Romboutsia, Bacteroides, and Clostridium_sensu_stricto_1 relative abundance values were significantly affected by berberine (Fig. 2b).

Berberine Improves DHEA-Induced PCOS
In addition, to find out the key discriminatory OTUs, the quasi-likelihood $F$ test was used here. A total of 26 differential OTUs between PCOS + HFD + BBR and PCOS + HFD groups were obtained with the cutoff value of $p$ value $<0.05$. Of these differential OTUs, a total of 10 OTUs were upregulated and 16 were downregulated. The differential bacteria taxa on phylum and genus levels between the 2 groups are displayed in Figure 3.

Besides, in order to infer the possible cooperative or competitive relationships between these differential gut bacterial microbiomes, correlation analysis was carried out. As shown in Figure 4, a total of 123 relational interactions were obtained with the threshold of $p$ value $<0.05$ and $|\mathrm{r}|>0.8$. Among these, a cooperative relationship was found between Ruminiclostridium_6 and Tyzzerella, and a competitive relationship existed between Alistipes and Family_XIII_AD3011_group.

\section{Berberine-Induced Metabolic Profiling Analysis}

After data preprocessing (online suppl. Fig. 1; see www.karger.com/doi/10.1159/000518040 for all online suppl. material), PCA, OPLS-DA, and PLS-DA were subsequently employed to reveal the clustering trends of each group. As shown in Figure 5, 2 samples (S4 and S5) showed serious deviation (Fig. 5a), and thus the 2 samples were removed. Then, the samples were divided into blocks suggesting that the 2 clusters were clearly divided (Fig. 5b), indicating 2 groups with different metabolic profiles. And, the results of OPLS-DA and PLS-DA show that samples were clustered closely together, which indicates that the experiment had good stability and repeat- 
a

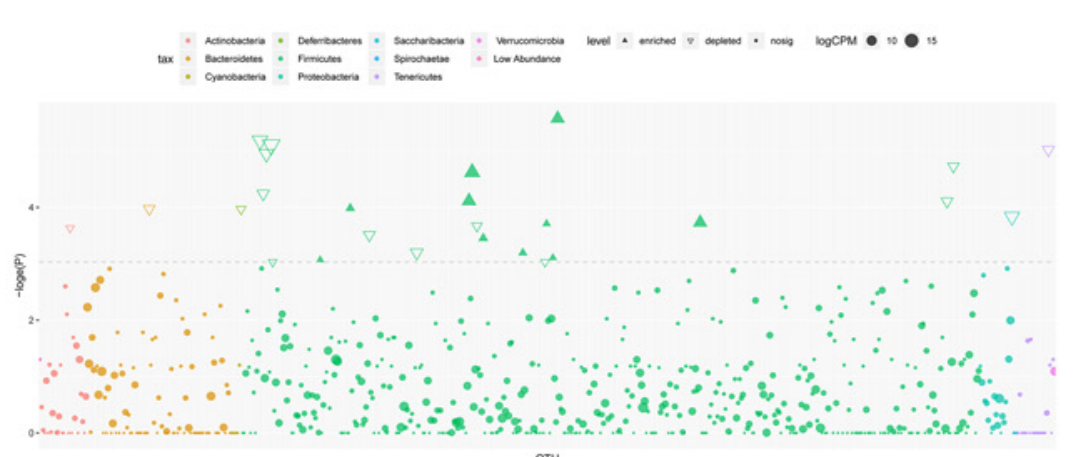

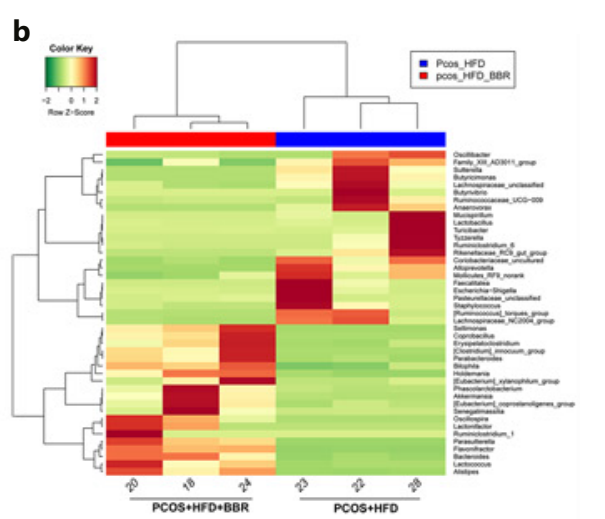

Fig. 3. The differential bacteria taxa between 2 groups. At the phylum level (a); at the genus level (b).

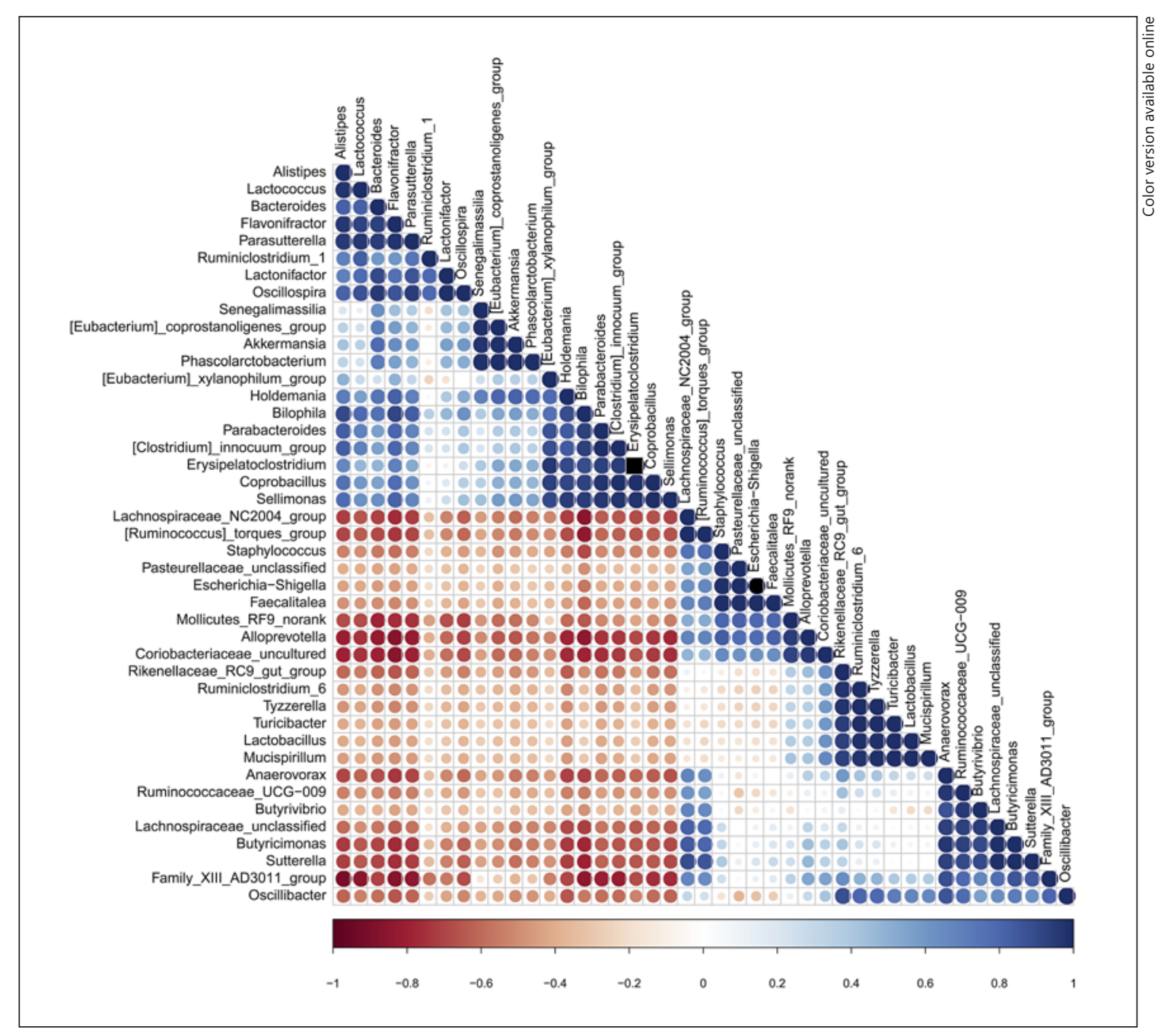

Fig. 4. The relationships between the differential gut microbiotas. 


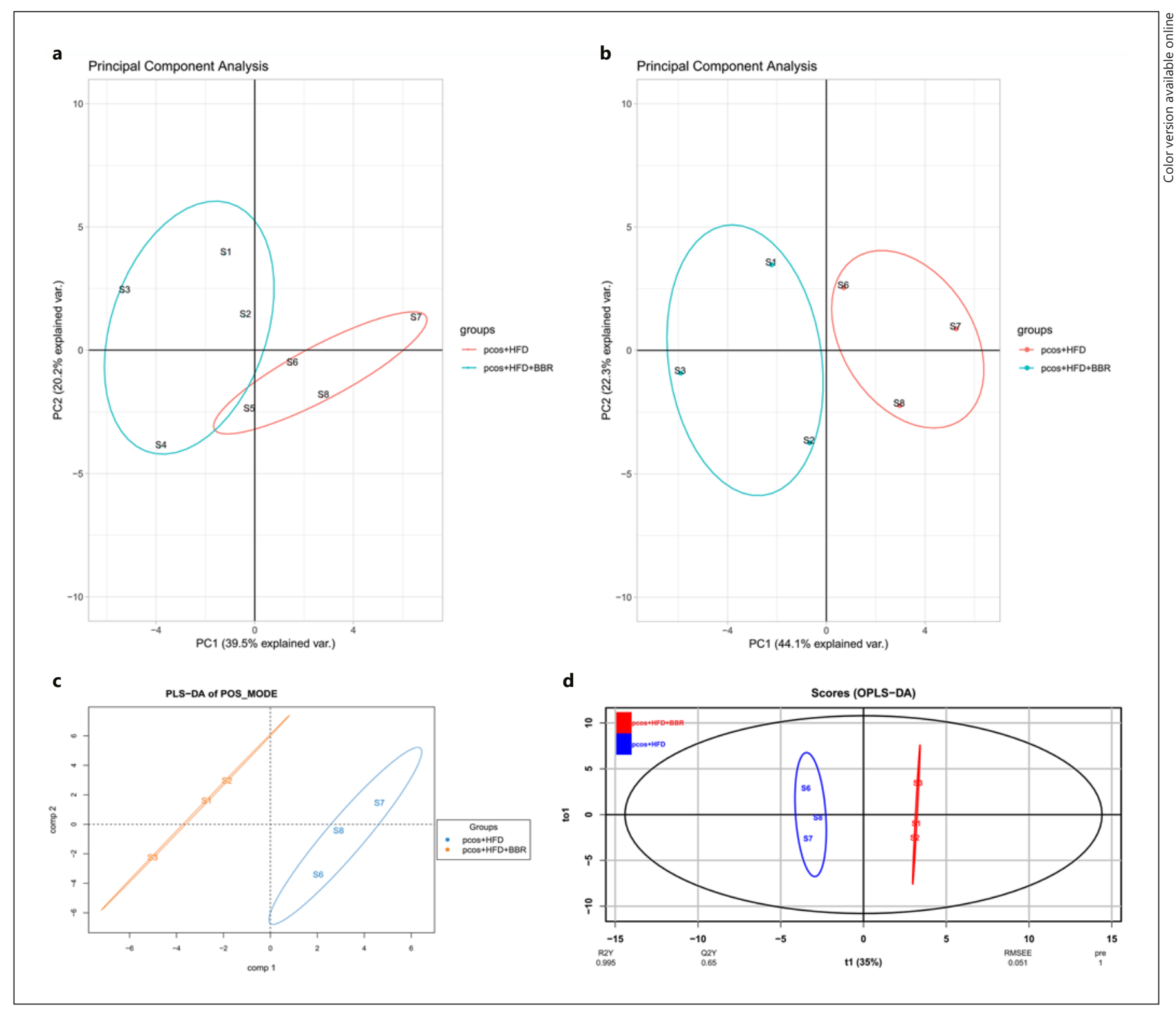

Fig. 5. Berberine alters metabolites in PCOS rats. PCA of all samples (a) and after removing 2 samples (S4 and S5) (b); OPLS-DA (c) and PLS-DA (d). PCOS, polycystic ovary syndrome; PCA, principal component analysis; OPLS-DA, orthogonal partial least-squares discriminant analysis.

ability (Fig. 5c, d). Three metabolites with the cutoff value $p$ value $<0.05$ and VIP $>1$ were screened between the 2 groups, including 1 upregulated (glutamine) and 2 downregulated (unsaturated acids, UFA $[\mathrm{CH}=\mathrm{CH}]$, and glucose) (Fig. 6a). The enrichment analysis showed that 22 Kyoto Encyclopedia of Genes and Genomes (KEGG) pathways, including type 2 diabetes mellitus, 2-component system, and $A B C$ transporters, were screened with the threshold of $p$ value $<0.05$ (Fig. 6b).

\section{Correlation Analysis}

As mentioned before, a total of 22 relationships were obtained, including 2 metabolites and 19 differential microbiotas (Fig. 6c). And, 3 microbiotas (Lachnospiraceae_ NC2004_group, Flavonifractor, and Parasutterella) were regulated by glucose and glutamine. 


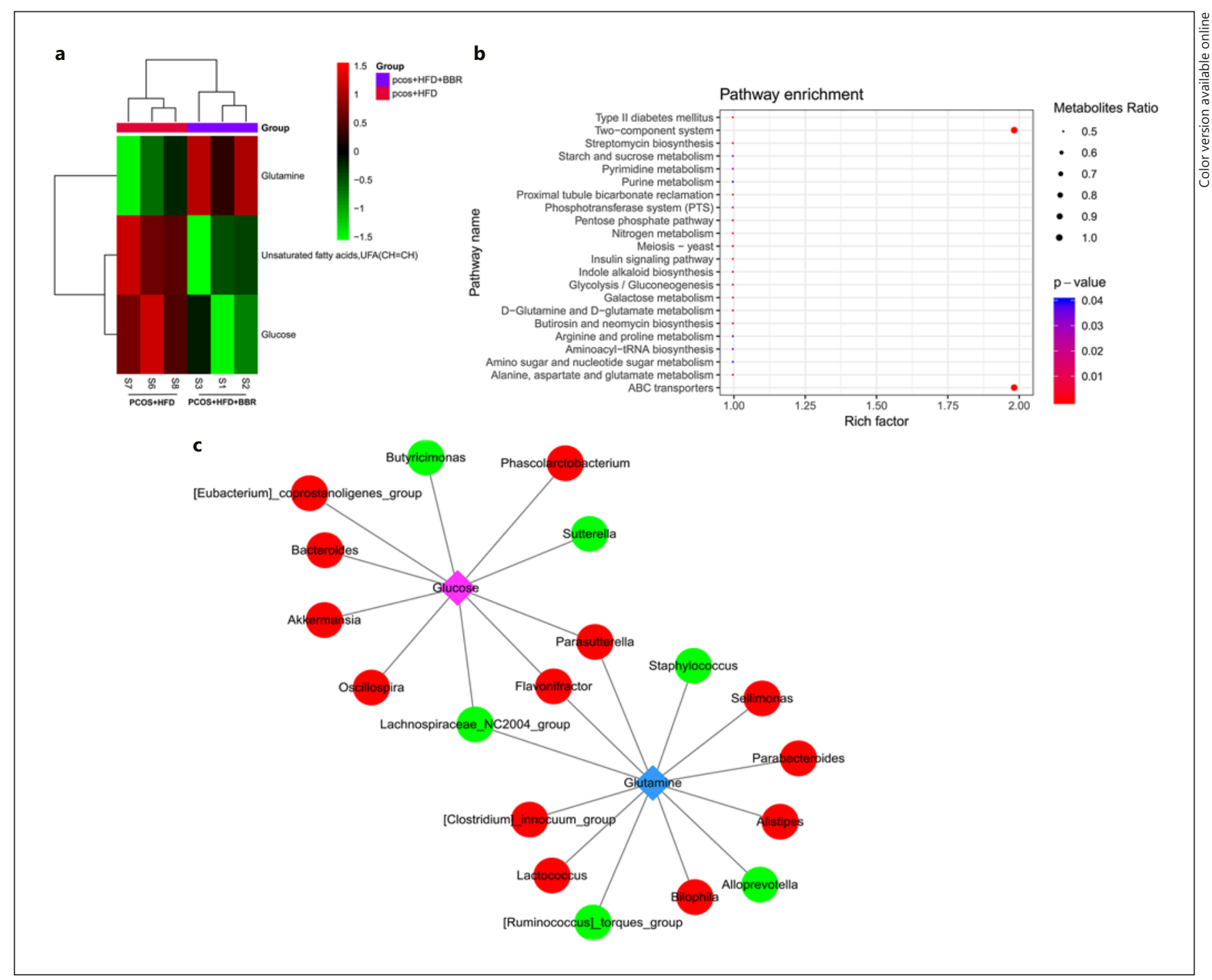

Fig. 6. The differential metabolites between 2 groups and correlation analysis between differential microbiotas and metabolites. Heatmap of differential metabolites. Red indicates upregulated, and green indicates downregulated (a); pathway enrichment analysis of differential metabolites. The size of the ball represents the metabolite ratio enriched in each term, and the color of the ball

\section{Discussion}

PCOS is an endocrine disease with complex and diverse manifestations of metabolic disorders [22, 23]. At present, drugs constitute the principal treatment for PCOS, and lifestyle adjustment is advocated to control diet, exercise, and weight $[24,25]$. Berberine, a major active component of the Chinese herbal medicines Rhizoma Coptidis, Cortex Phellodendri, and Cortex Berberidis, has represents the number of the $p$ value (b); correlation analysis between differential microbiotas and metabolites. The red nodes indicate upregulated microbiotas, and green nodes indicate downregulated microbiotas. The pink diamond indicates upregulated metabolite, and blue diamond indicates downregulated metabolite $(\mathbf{c})$.

been prescribed for the treatment of diarrhea, metabolic disorders, and infertility [26]. There is recent evidence indicating that berberine offers promise for treating PCOSIR [27]. In this study, Firmicutes and Bacteroidetes were changed at the phylum level, and Romboutsia, Bacteroides, and Clostridium_sensu_stricto_1 were changed at the genus level. In addition, a total of 26 differential OTUs and 3 metabolites (glutamine, unsaturated acids, UFA [CH = $\mathrm{CH}$ ], and glucose) between 2 groups were obtained. More- 
over, these metabolites were mainly involved in the type 2 diabetes mellitus, 2-component system, and ABC transporter KEGG pathways. And, 3 microbiotas (Lachnospiraceae_NC2004_group, Flavonifractor, and Parasutterella) were regulated by glucose and glutamine.

As we all know, the ratio of Firmicutes to Bacteroidetes is considered as a key indicator to measure the state of gut microbiota $[28,29]$. In this study, Firmicutes were decreased and Bacteroidetes were increased at the phylum level after berberine treatment, which means that the berberine improved the structure of gut microbiota. Besides, Kelley et al. [30] suggested that Bacteroidales and Clostridiales were changed in a letrozole-induced PCOS rat model, which were consistent with our results. In addition, a total of 26 differential OTUs were dysregulated; one possible explanation for the changes we observed in the gut microbiome is that berberine had a direct effect on the gut microbiome.

PCOS is a complex endocrinopathy, which includes numerous abnormalities and influences some metabolic pathways. Thus, the metabolomic analysis was performed, and 3 metabolites, including glutamine, unsaturated acids, UFA $[\mathrm{CH}=\mathrm{CH}]$, and glucose, were changed after berberine treatment. Glutamine is the most abundant amino acid in the body, with antioxidant and anti-inflammatory properties [31,32]. Numerous studies indicated that oxidative stress and chronic inflammation might have an important effect on the pathophysiology of PCOS [33-35]. In addition, glucose is capable of inciting oxidative stress and an inflammatory response from mononuclear cells of women with PCOS [7]. Herein, glutamine was increased, and glucose and unsaturated acids, UFA $[\mathrm{CH}=\mathrm{CH}]$, were decreased after berberine treatment, illustrating that berberine might be effective in improving the pathological condition in PCOS. Besides, the enrichment analysis showed that these metabolites were mainly involved in the type 2 diabetes mellitus, 2-component system, and $\mathrm{ABC}$ transporter KEGG pathways. Due to the high prevalence of type 2 diabetes in women with this syndrome and their relatives, PCOS should be considered a marker of family pathology, a pathway to type 2 diabetes [36]. ABC transporters are primary active membrane proteins that translocate solutes across lipid bilayers [37]. Members of the ABC transporter family, such as ABCA1, have been shown to control cellular lipid metabolism. Changes happening in the ATP binding cassette transporter 1 (ABC1) gene encoding a protein regulating entry and exit from the cell membrane may contribute to dyslipidemia in patients with PCOS [38]. However, few studies of the 2-component system pathway on the PCOS have been reported. Taken to- gether, we suspected that berberine might be effective in improving the pathological condition in PCOS by regulating these metabolites via type 2 diabetes mellitus, 2-component system, and ABC transporter KEGG pathways.

Besides, in this study, the correlation analysis showed that glutamine was regulated by 12 microbiotas, and glucose was regulated by 10 microbiotas. Increasing evidence implied that gut microbiome functions by regulating metabolites that can directly or indirectly affect host physiology $[39,40]$. In addition, dysbiosis of gut microbiota theory revealed that gut microbiome dysbiosis causes a breakdown in gut mucosal permeability, passage of gut microbiome endotoxin into the systemic circulation, inflammation initiating IR, and an increase in testosterone production - PCOS phenotype [19]. Moreover, glutamine is an important metabolite involved in maintaining gut wall barrier integrity [41], and glutamine was increased after berberine treatment in this study. Therefore, we speculated that one possible mechanism for berberine action might be via modulating gut wall barrier integrity to prevent gut microbiome endotoxin into the systemic circulation.

However, this study has some limitations. The sample size involved in this study is relatively small, and a large sample size should be used to carry out further study. In addition, relevant experiments need to be performed to verify the obtained results from this study, and in-depth functional studies are needed.

\section{Conclusion}

These results confirmed that the gut microbiome is altered and contributes to performance by interacting with metabolism under berberine application. Collectively, our results provide a guide for future research in the development of medical treatments for PCOS.

\section{Acknowledgments}

We would like to thank LetPub (www.letpub.com) for providing linguistic assistance during the preparation of the manuscript.

\section{Statement of Ethics}

All the animal experiments were conducted in strict accordance with the animal ethics standards and with full ethical approval of the Ethics Committee of Obstetrics and Gynecology Hospital of Fudan University (No. [2015] 45). 


\section{Conflict of Interest Statement}

The authors declare that they have no conflicts of interest.

\section{Funding Sources}

This study was supported by the Natural Science Foundation from the Science and Technology Commission of Shanghai $\mathrm{Mu}-$ nicipality (Grant No. 17ZR1403100 to Xue-Lian Li).

\section{Author Contributions}

X.L. contributed to conception and design of the research; H.S. was involved in acquisition of data; H.S. and X.X. analyzed and interpreted the data; D.Y. performed statistical analysis; X.L. obtained funding; H.S. and X.X. drafted the manuscript; X.L. revised the manuscript for important intellectual content. All authors read and approved the final manuscript.

\section{References}

1 Azziz R, Woods KS, Reyna R, Key TJ, Knochenhauer ES, Yildiz BO. The prevalence and features of the polycystic ovary syndrome in an unselected population. J Clin Endocrinol Metab. 2004 Jun;89(6):2745-9.

2 Jiang X, Ding C, Wang C, Yu J, Hua Z, Chen $\mathrm{X}$, et al. Association of paraoxonase 1 gene polymorphisms and polycystic ovarian syndrome susceptibility: a systematic review and meta-analysis. Gynecol Obstet Invest. 2020; 85(2):167-77.

3 Yang SH, Hur YS, Yoon SH, Jung JH, Lim JH, Ko Y. A comparison of embryonic development and clinical outcomes between in vitro oocytes maturation using micro-nibration system and in vivo oocytes maturation in polycystic ovarian syndrome patients. Gynecol Obstet Invest. 2020;85(3):252-8.

4 Qi Y, Yin Q, Gu J, Liu Y, Sun Q, Cao Y, et al. Elevated circulating levels of carnal ENST00000550337.1 Are associated with polycystic ovary syndrome in Chinese women. Gynecol Obstet Invest. 2021 Feb 18;86(12):155-61.

5 Li R, Zhang Q, Yang D, Li S, Lu S, Wu X, et al. Prevalence of polycystic ovary syndrome in women in China: a large community-based study. Hum Reprod. 2013;28(9):2562-9.

6 Diamanti-Kandarakis E, Kouli C, Tsianateli $\mathrm{T}$, Bergiele A. Therapeutic effects of metformin on insulin resistance and hyperandrogenism in polycystic ovary syndrome. Eur J Endocrinol. 1998;138(3):269.

7 González F. Inflammation in polycystic ovary syndrome: underpinning of insulin resistance and ovarian dysfunction. Steroids. 2012; 77(4):300-5.

8 Dragamestianos C, Messini CI, Antonakis PT, Zacharouli K, Kostopoulou E, Makrigiannakis A, et al. The effect of metformin on the endometrium of women with polycystic ovary syndrome. Gynecol Obstet Invest. 2019;84(1):35-44.

9 Tan X, Li S, Chang Y, Fang C, Liu H, Zhang $\mathrm{X}$, et al. Effect of metformin treatment during pregnancy on women with PCOS: a systematic review and meta-analysis. Clin Invest Med. 2016 Sep 11;39(4):E120-31.
10 Taylor CE, Greenough WB. Control of diarrheal diseases. Annu Rev Public Health. 1989; 10(1):221

11 Wang Y, Shou JW, Li XY, Zhao ZX, Fu J, He $\mathrm{CY}$, et al. Berberine-induced bioactive metabolites of the gut microbiota improve energy metabolism. Metabolism. 2017;70:72.

12 Zhang X, Zhao Y, Zhang M, Pang X, Xu J, Kang C, et al. Structural changes of gut microbiota during berberine-mediated prevention of obesity and insulin resistance in high-fat diet-fed rats. PLos One. 2012;7(8):e42529.

13 Zhang Y, Sun J, Zhang YJ, Chai QY, Zhang K, $\mathrm{Ma} \mathrm{HL}$, et al. The effect of berberine on insulin resistance in women with polycystic ovary syndrome: detailed statistical analysis plan (SAP) for a multicenter randomized controlled trial. Trials. 2016;17(1):512.

14 Liu D, Zhang Y, Liu Y, Hou L, Li S, Tian H, et al. Berberine modulates gut microbiota and reduces insulin resistance via the TLR4 signaling pathway. Exp Clin Endocrinol Diabetes. 2018;126(8):51320

15 Wu XK, Wang YY, Liu JP, Liang RN, Xue HY, $\mathrm{Ma} \mathrm{HX}$, et al. Randomized controlled trial of letrozole, berberine, or a combination for infertility in the polycystic ovary syndrome. Fertil Steril. 2016 Sep 1;106(3):757-65.e1.

16 Mayer EA. Gut feelings: the emerging biology of gut-brain communication. Nat Rev Neurosci. 2011;12(8):453-66.

17 Guo Y, Qi Y, Yang X, Zhao L, Wen S, Liu Y, et al. Association between polycystic ovary syndrome and gut microbiota. PLoS One. 2016;11(4):e0153196.

18 Bowe JE, Franklin ZJ, Hauge-Evans AC, King AJ, Persaud SJ, Jones PM. Metabolic phenotyping guidelines: assessing glucose homeostasis in rodent models. J Endocrinol. 2014 Sep;222(3):G13-25.

19 Tremellen K, Pearce K. Dysbiosis of gut microbiota (DOGMA): a novel theory for the development of polycystic ovarian syndrome. Medical hypotheses. 2012;79(1):104-12.

20 Hao L, Jia X, Yu Q, Zhang C, Kang J. High-fat diet induces significant metabolic disorders in a mouse model of polycystic ovary syndrome. Biol Reprod. 2014;91(5):127-7.
21 Shen $\mathrm{H}-\mathrm{R}, \mathrm{Xu}$ X, Li X-L. Berberine exerts a protective effect on rats with polycystic ovary syndrome by inhibiting the inflammatory response and cell apoptosis. Reprod Biol Endocrinol. 2021;19(1):3.

22 Yang K, Zeng L, Bao T, Ge J. Effectiveness of omega-3 fatty acid for polycystic ovary syndrome: a systematic review and meta-analysis. Reprod Biol Endocrinol. 2018;16(1):27.

23 Kim SH, Liu M, Jin HS, Park S. High genetic risk scores of ASIC2, MACROD2, CHRM3, and $\mathrm{C} 2$ orf83 genetic variants associated with polycystic ovary syndrome impair insulin sensitivity and interact with energy intake in Korean women. Gynecol Obstet Invest. 2019; 84(3):225-36.

24 Bargiota A, Diamanti-Kandarakis E. The effects of old, new and emerging medicines on metabolic aberrations in PCOS. Ther Adv Endocrinol Metab. 2012;3(1):27-47.

25 Orostica L, Poblete C, Romero C, Vega M. Pro-inflammatory markers negatively regulate IRS1 in endometrial cells and endometrium from women with obesity and PCOS. Reprod Sci. 2020 Jan;27(1):290-300.

26 Feng X, Sureda A, Jafari S, Memariani Z, Tewari D, Annunziata G, et al. Berberine in cardiovascular and metabolic diseases: from mechanisms to therapeutics. Theranostics. 2019;9(7):1923-51.

27 Li MF, Zhou XM, Li XL. The effect of berberine on polycystic ovary syndrome patients with insulin resistance (PCOS-IR): a metaanalysis and systematic review. Evid Based Complement Alternat Med. 2018;2018: 2532935.

28 Foster JA, McVey Neufeld KA. Gut-brain axis: how the microbiome influences anxiety and depression. Trends Neurosci. 2013;36(5): 305-12.

29 Wong ML, Inserra A, Lewis MD, Mastronardi CA, Leong L, Choo J, et al. Inflammasome signaling affects anxiety- and depressive-like behavior and gut microbiome composition. Mol Psychiatry. 2016 Jun;21(6):797-805.

30 Kelley ST, Skarra DV, Rivera AJ, Thackray VG. The gut microbiome is altered in a letrozole-induced mouse model of polycystic ovary syndrome. PLoS One. 2016;11(1) e0146509. 
31 Raizel R, Leite JS, Hypólito TM, Coqueiro AY, Newsholme P, Cruzat VF, et al. Determination of the anti-inflammatory and cytoprotective effects of l-glutamine and l-alanine, or dipeptide, supplementation in rats submitted to resistance exercise. Br J Nutr. 2016 Aug; 116(3):470-9.

32 Nemati A, Alipanah-Moghadam R, Molazadeh L, Naghizadeh Baghi A. The effect of glutamine supplementation on oxidative stress and matrix metalloproteinase 2 and 9 after exhaustive exercise. Drug Des Devel Ther. 2019; 13:4215-23.

33 Meier RK. Polycystic ovary syndrome. Nurs Clin North Am. 2018 Sep;53(3):407-20.

34 Mohammadi M. Oxidative stress and polycystic ovary syndrome: a brief review. Int J Prev Med. 2019;10:86.
35 Emidio GD, Placidi M, Rea F, Rossi G, Falone $\mathrm{S}$, Cristiano L, et al. Methylglyoxal-dependent glycative stress and deregulation of SIRT1 functional network in the ovary of PCOS mice. Cells. 2020 Jan 14;9(1):209.

36 Sir-Petermann T. Polycystic ovary syndrome, a pathway to type 2 diabetes. Nutrition. 2005 Nov-Dec;21(11-12):1160-3

37 Oram JF, Lawn RM. ABCA1. The gatekeeper for eliminating excess tissue cholesterol. J Lipid Res. 2001 Aug;42(8):1173-9.

38 Karadeniz M, Erdoğan M, Ayhan Z, Yalcin M, Olukman M, Cetinkalp S, et al. Effect Of G2706A and G1051A polymorphisms of the $\mathrm{ABCA} 1$ gene on the lipid, oxidative stress and homocystein levels in Turkish patients with polycystıc ovary syndrome. Lipids Health Dis. 2011 Oct 28;10:193.
39 Putignani L, Del Chierico F, Vernocchi P, Cicala M, Cucchiara S, Dallapiccola B. Gut microbiota dysbiosis as risk and premorbid factors of IBD and IBS along the childhoodadulthood transition. Inflamm Bowel Dis. 2016 Feb;22(2):487-504

40 Tang WHW, Li DY, Hazen SL. Dietary metabolism, the gut microbiome, and heart failure. Nat Rev Cardiol. 2019 Mar;16(3):137-54.

41 Chen K, Okuma T, Okamura K, Torigoe Y, Miyauchi Y. Glutamine-supplemented parenteral nutrition improves gut mucosa integrity and function in endotoxemic rats. JPEN Parenter Enteral Nutr. 1994 Mar-Apr;18(2): $167-71$. 